Dahlia hybrida تأثير الرش بالباكلوبترازول والعناصر الغذائية الصغرى في نمو نباتات الداليا صنف Edinburgh باستخدام طريقتين للإكثار

r- بفات الجذور المتدرنة

عمار عمر الأطرقجي

هالة عبدالرحمن عبد القادر

قسم البستنة و هندسة الحدائق/ كلية الزر اعة و الغابات / جامعة الموصل الرط

الخلاصة

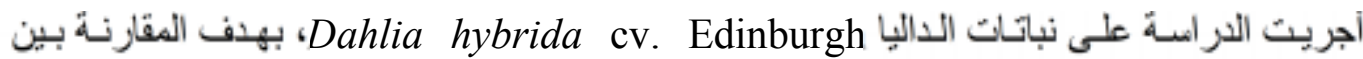

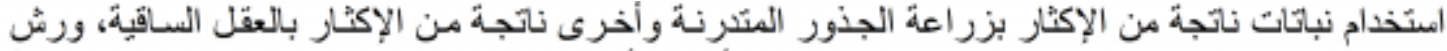

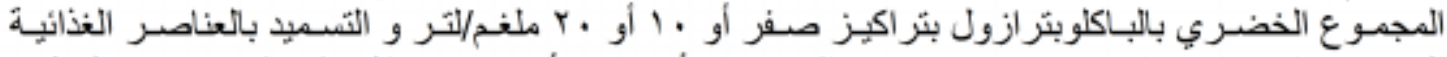

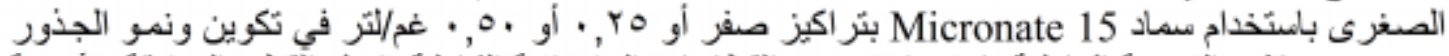

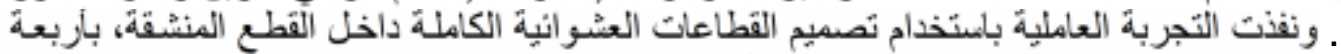

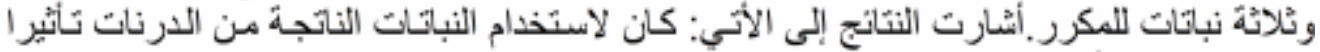

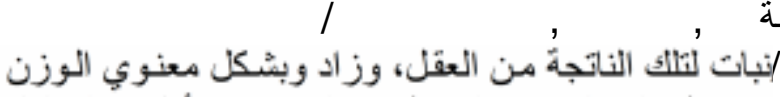
معنوياً في الحصول على أكبر عدد للجذور المتدرنة

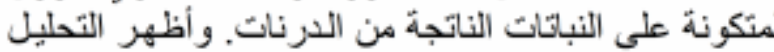

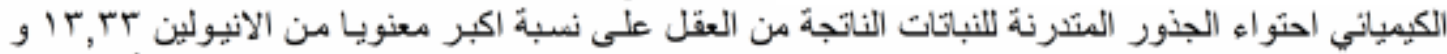

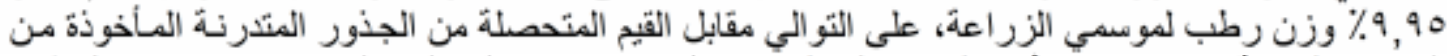

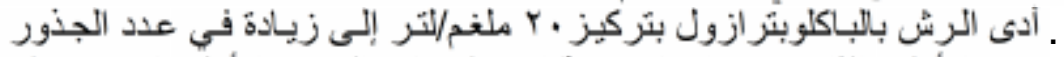

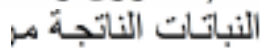

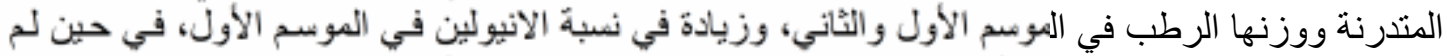

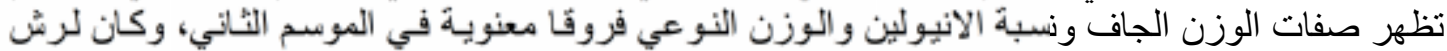

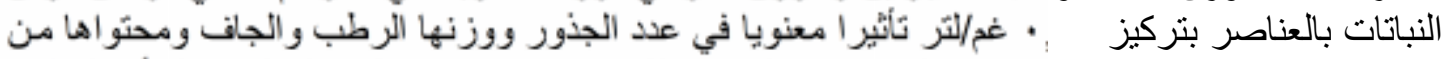

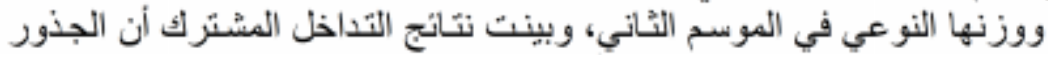

نيولين

\title{
المقدمة
}

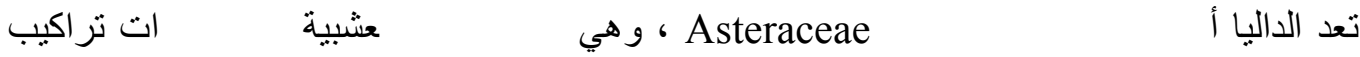
حقيقية مشابه للجذور الاعتيادية في تركيبها الشكلي

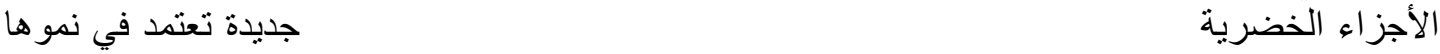

والتشريحي هذه الجذور تكون محولة Biennial ي على الغذاء المخزون في الجذور القديمة و التي سوف تضمحل خلال موسم النمو لتك

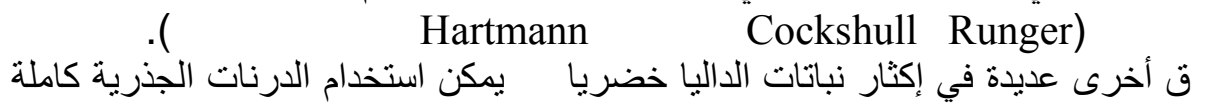

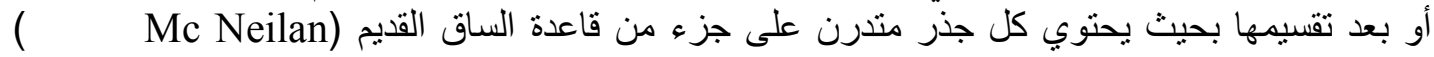
ساقية ناتجة من نمو الفروع على منطقة التاج

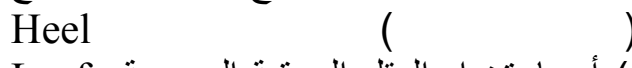
Leaf- أو باستخدام العقل الورقية البرعية ( Rowlands)

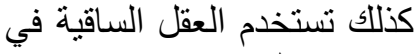
بعد وصولها ( Hartmann) bud cutting تخزن الداليا في درناتها الجذرية السكريات بشكل إنيولين Inulin و الذي هو

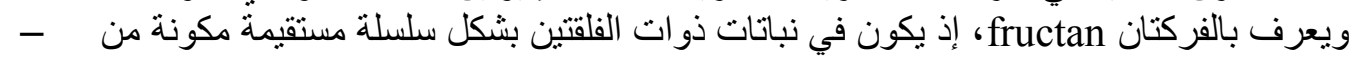

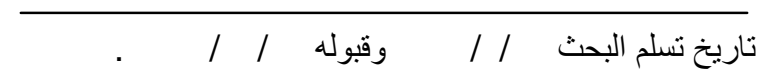

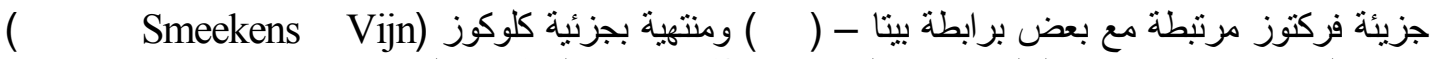

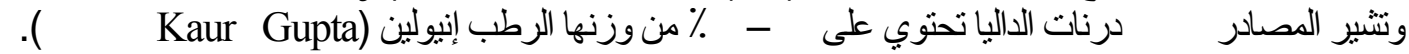



( ) ( )
(ISSN 1815-316X)
مجلة زر اعة الر افدين
الداليا تستجيب لمعوقات
تكوين درنات نبات الداليا Dahlia variabilis 'sneezy' كون الزير
يد من الدراسات () Mess Moser
( ) Hess كون البر عم الزهري و

Alar

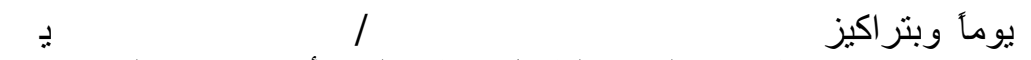

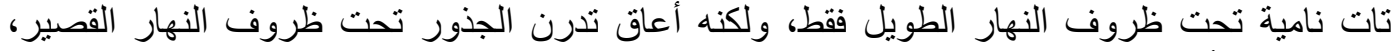

تأثثير

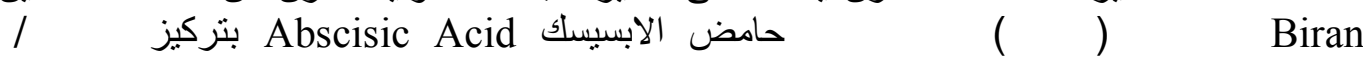
(SADH) Succinic acid-2, 2-di-methylhydrazid النامية تحت ظروف النهار الطويل من تكوين الدرنات والتي وصل وزنها

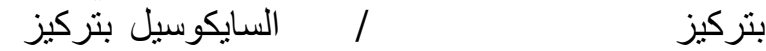

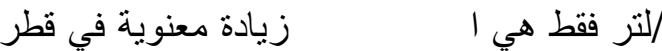

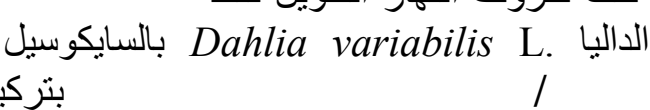
| ولكن المعاملة بالسايكوسيل بتركيز لها.

بنز

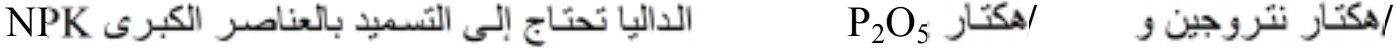

Cl Cu Zn Fe فإنها

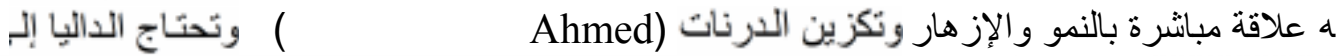

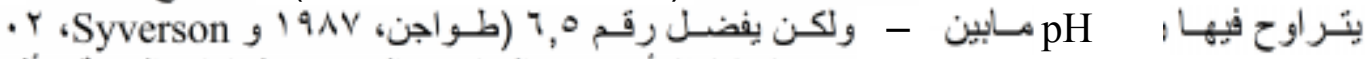

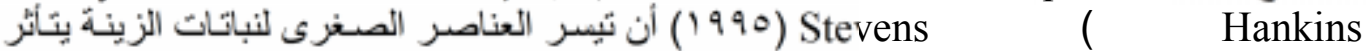

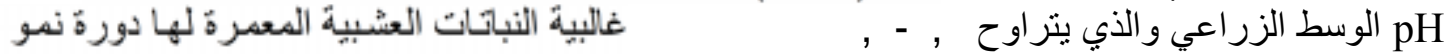

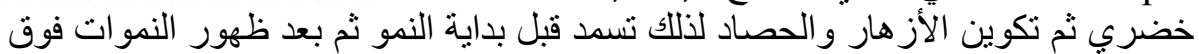

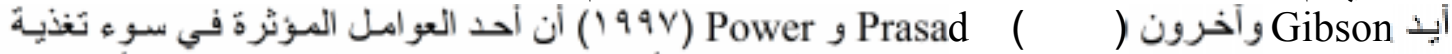

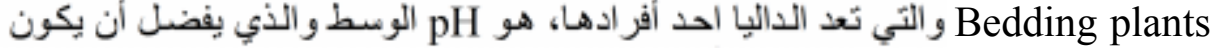

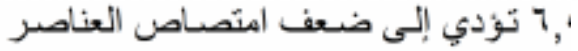

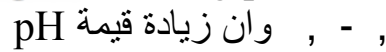

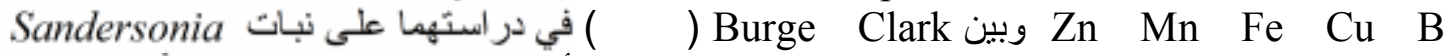
pH aurantiaca

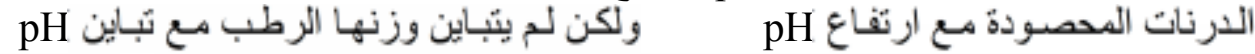

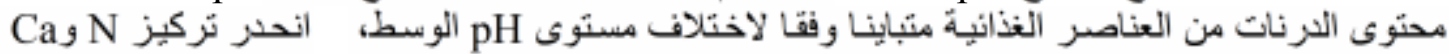

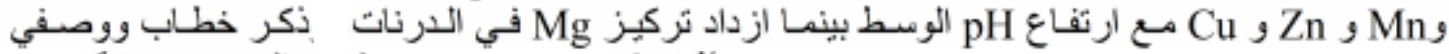

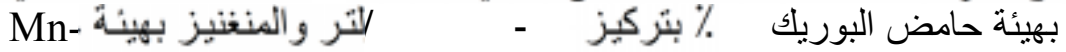

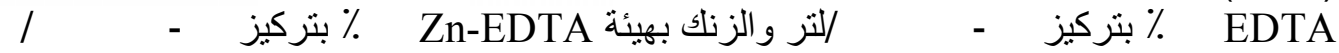

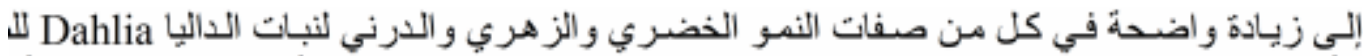

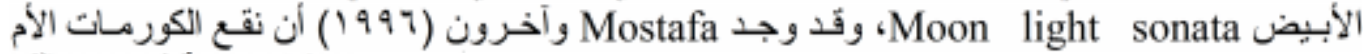

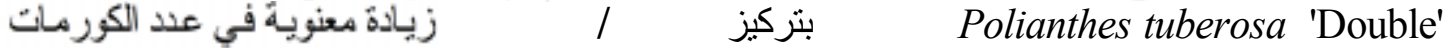

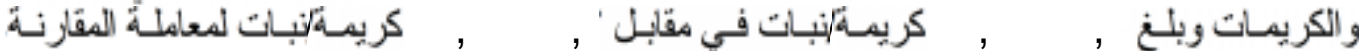

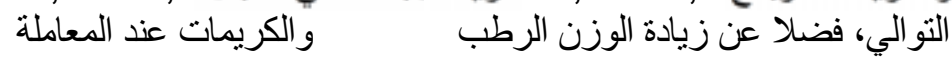

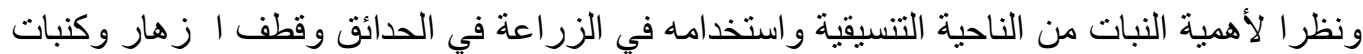

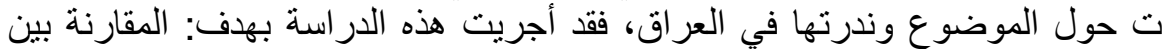

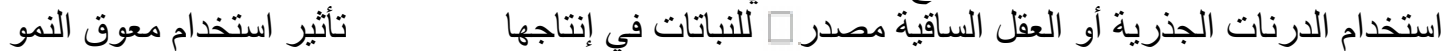
تقييم محتوى الدرنات الجذرية من التوف التون

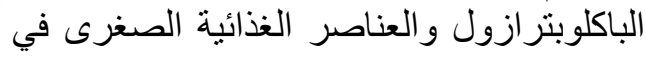
الانيولين بفعل المعاملات موضول والعنر الفذانة الدراسة.

المواد البحث وطرائقه

أجريت التجربة في مدينة الموصل/ لي لمول

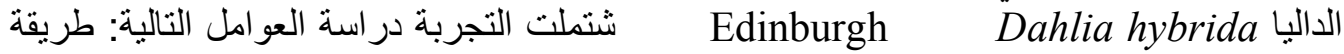


المقارنة بين نباتات ناتجة من زراعة درنات جذرية مباشرة وأخرى ناتجة من عقل ساقية طرفية تم

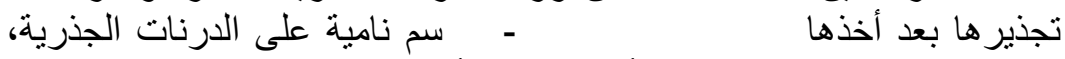

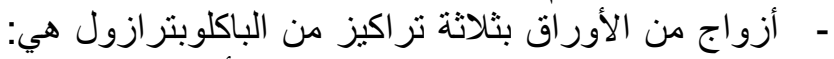

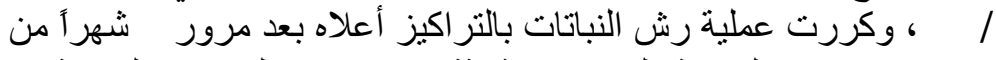

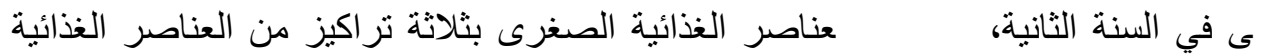

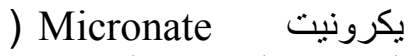

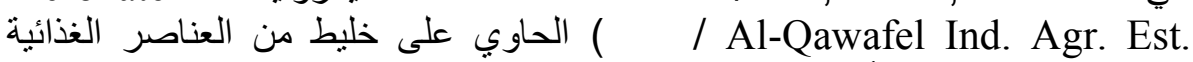
Micronutrient Mix EDTA ثيلين ثنائي الأمين رباعي حامض الخليك وحامض الستريك

, $\quad \mathrm{Cu}, \quad \mathrm{Mn}$, $\quad \mathrm{Fe}$, إذ يحتوي على (بنسبة مئوية\%) Citric Acid Chelation

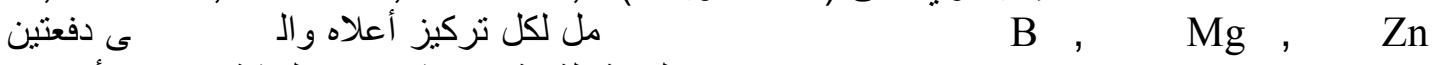

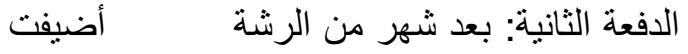

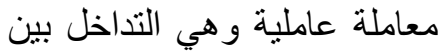

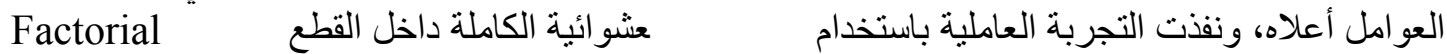
Experiment Within Split Plot in Randomized Complete Block Design

$$
\begin{aligned}
& \text { استخدمت درنات جذرية متجانسة من إنتاج شركة أبصال غوطة دمشق/سوريا وزنها }
\end{aligned}
$$

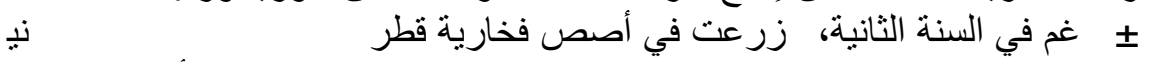

أسابيع من عن

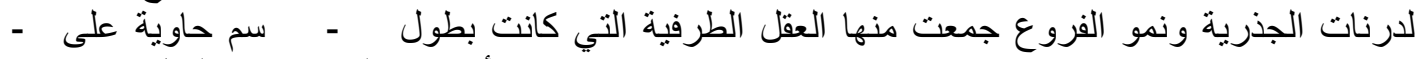

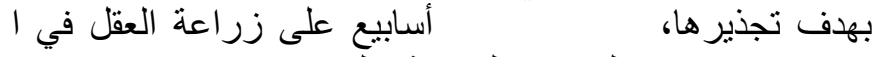

$$
\text { . }
$$

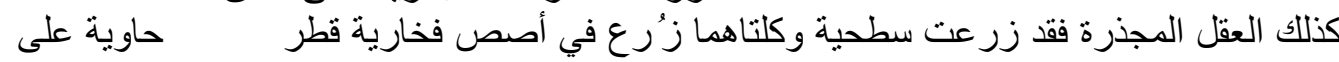

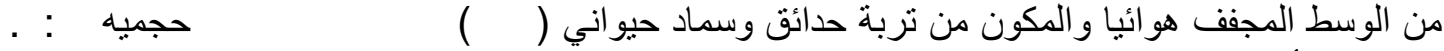

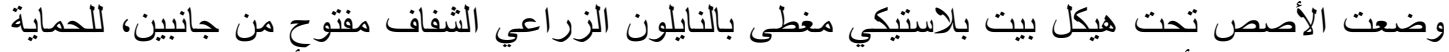

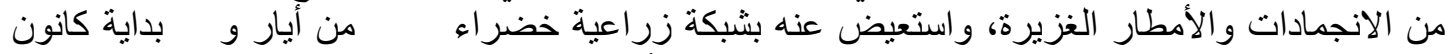

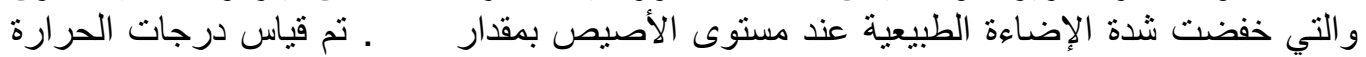

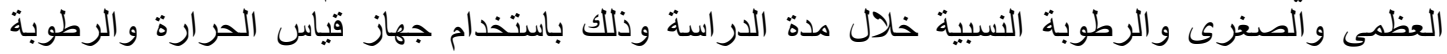
. تم إضافة السماد النتروجيني

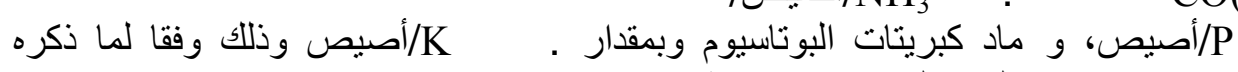

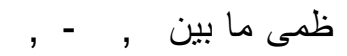

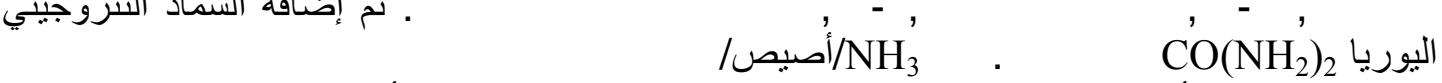

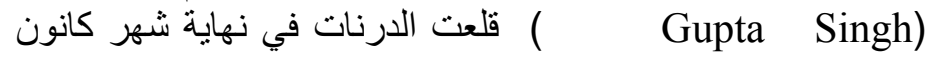

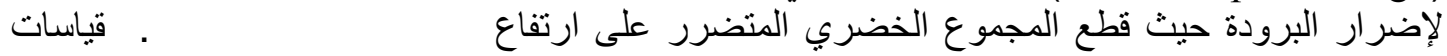

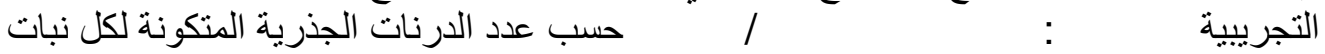

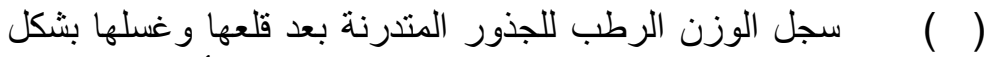
جيد من الأتربة العالقة بها، ثم جفقت في فرن كهربائي على درجة حرارة حسب المعادلة التالية:

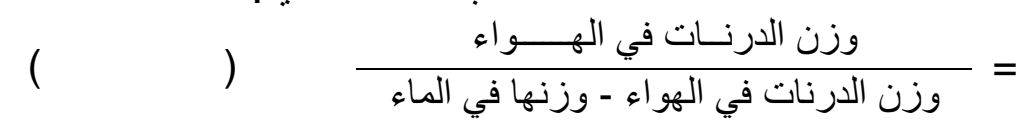

تركيز الانيولين في الجذور المتدرنة( ) وذلك باعتماد طريقة تحليلية، بتقدير الفركتوز في العينة النباتية

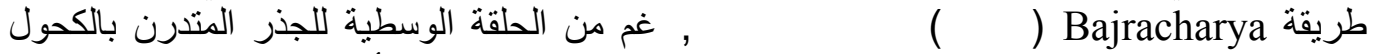

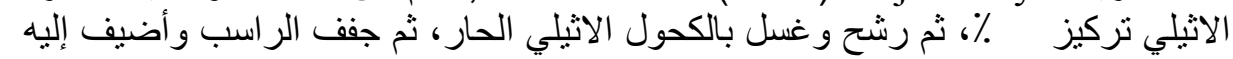

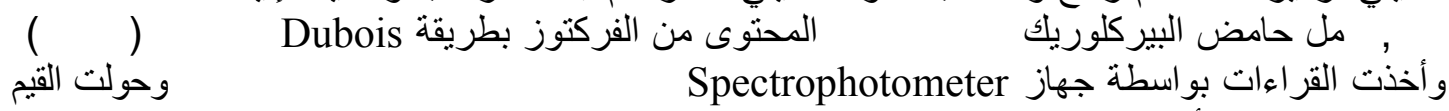
نسبة مئوية، وأجري تحليل التباين 


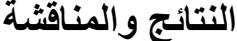

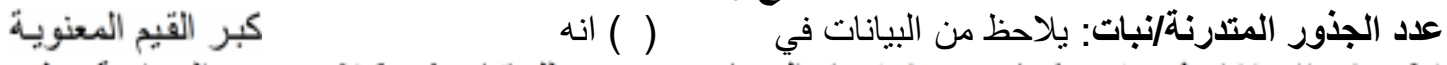

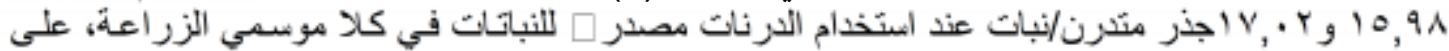

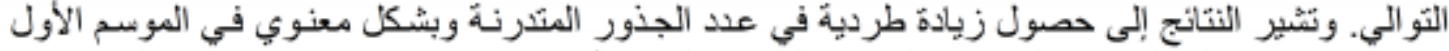

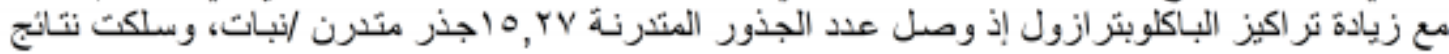

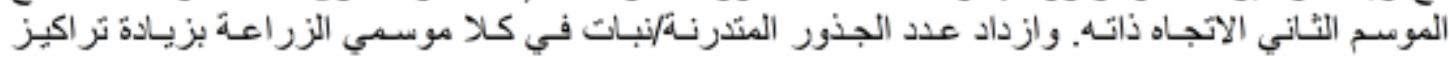

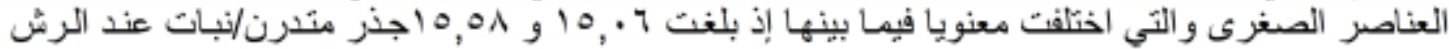

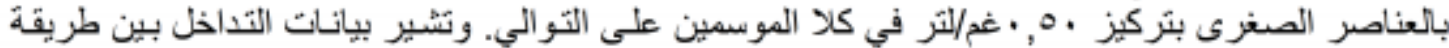

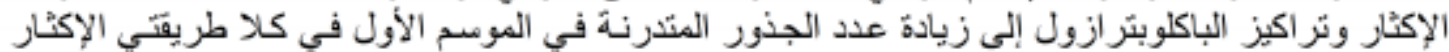

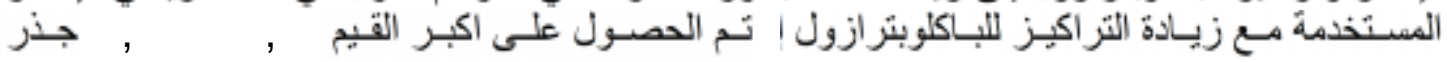

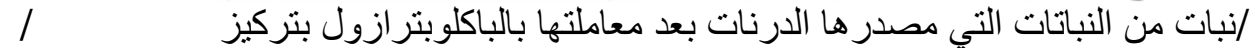

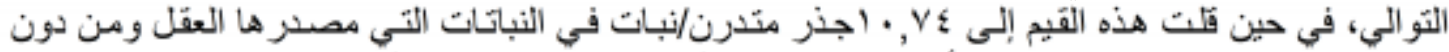

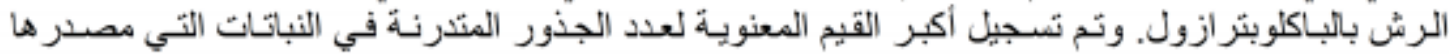

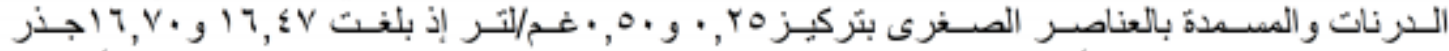

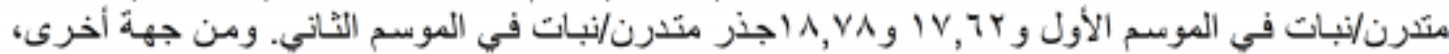

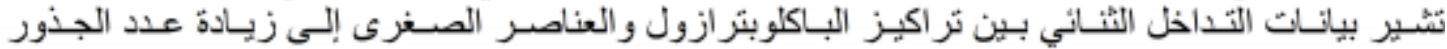

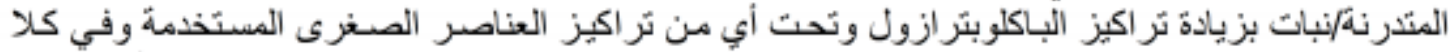

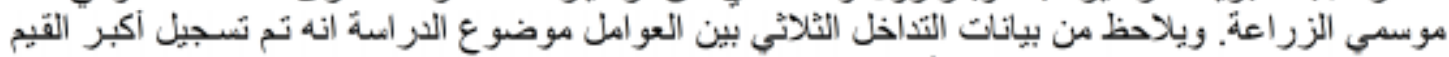

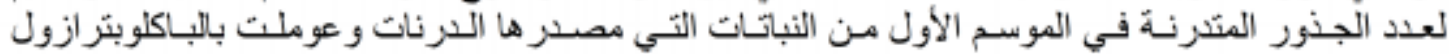

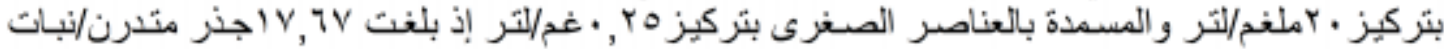

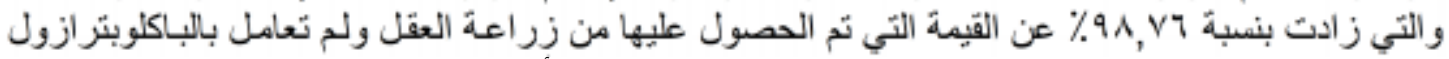

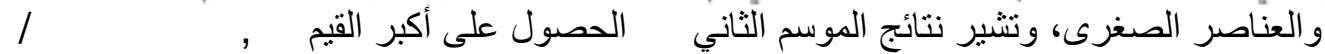

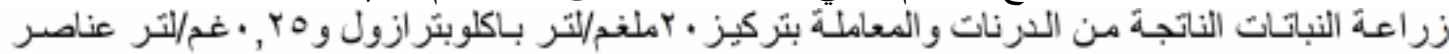

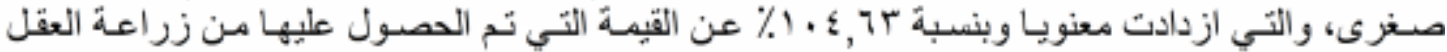

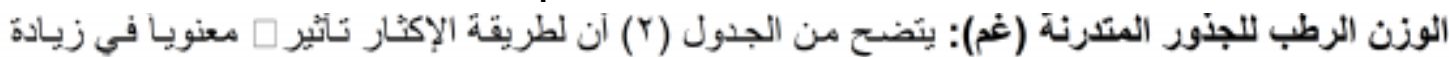

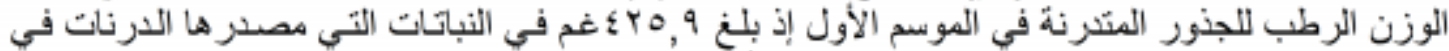

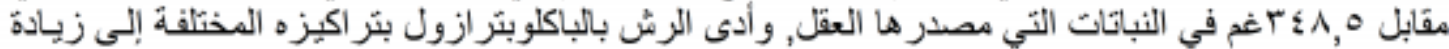

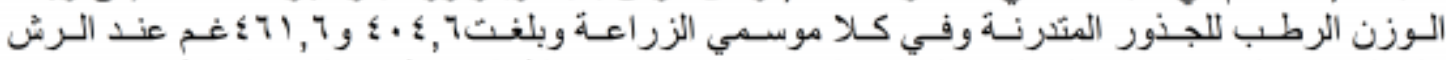

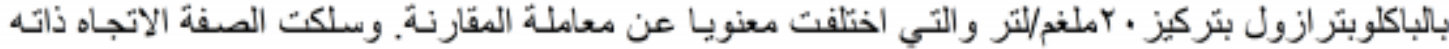

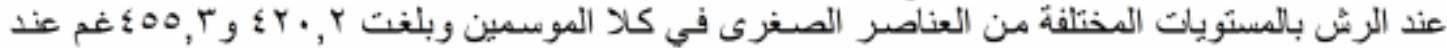

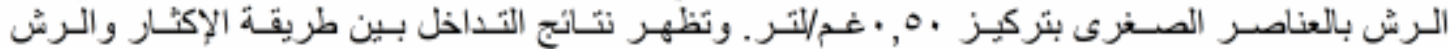

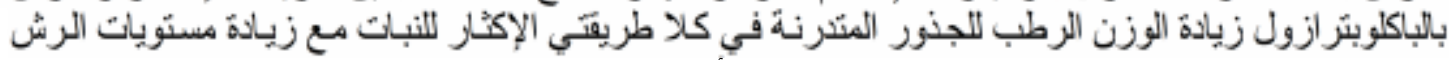

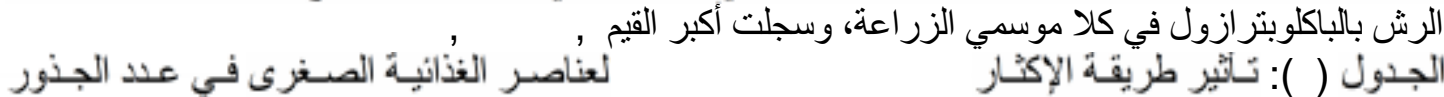
/ النبات لموسمي الزر اعة لنباتات الداليا.

\begin{tabular}{|c|c|c|c|c|c|c|}
\hline \multirow{2}{*}{ تأثير طريقة } & \multirow[t]{2}{*}{ تداخل طريقة } & \multicolumn{3}{|c|}{ تراكيز العناصر الصغرى ( / / ) } & \multirow{2}{*}{ تر اكيز } & \multirow{2}{*}{ طريقة } \\
\hline & & , & , & & & \\
\hline \multirow{3}{*}{, } & , & $-\quad$, & - & - & & \multirow{3}{*}{ درنات جذرية } \\
\hline & , & 1 & 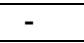 & $\rightarrow-$ & & \\
\hline & , & , & , & 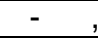 & & \\
\hline \multirow{3}{*}{, } & $\rightarrow$ & , & , & , & & \multirow{3}{*}{ عقل ساقية } \\
\hline & , & , & , & , & & \\
\hline & , & $-\quad$, & $9 \rightarrow$ & , & & \\
\hline \multirow{2}{*}{\multicolumn{2}{|c|}{ تأثير }} & , & , & , & & \multirow{2}{*}{ تداخل طريقة } \\
\hline & & & & هـ & & \\
\hline
\end{tabular}



()$\quad(\quad)$
(ISSN 1815-316X)
مجلة زر اعة الر افدين

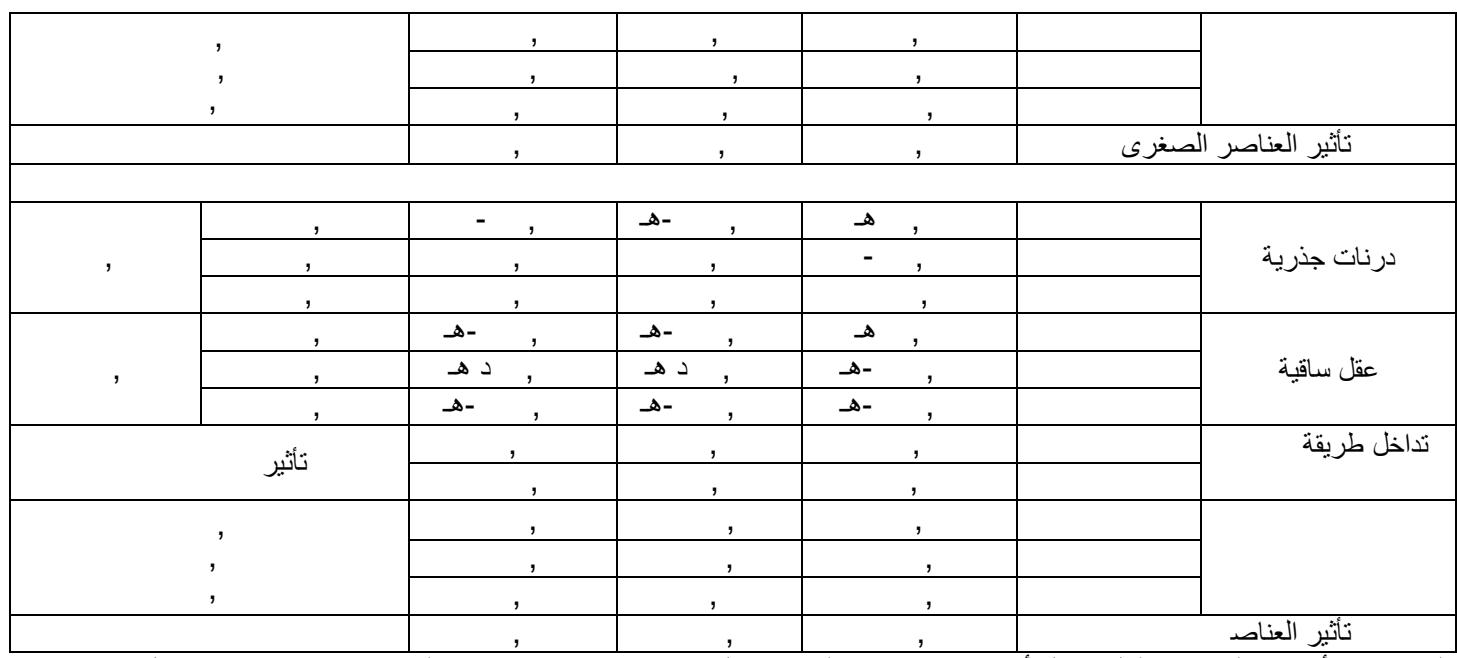

القيم ذات الأحرف المتشابهة لكل عامل أو تداخلاتها لا تختلف معنويأ حسب اختبار دنكن متعدد الحدود تحت مستوى احتمال

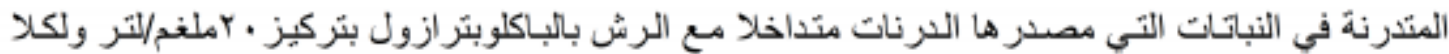

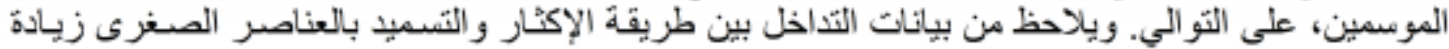

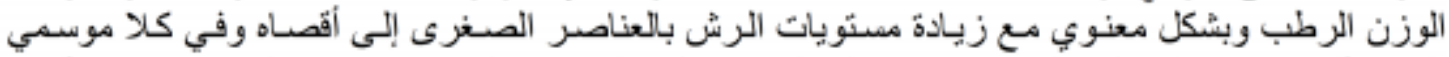

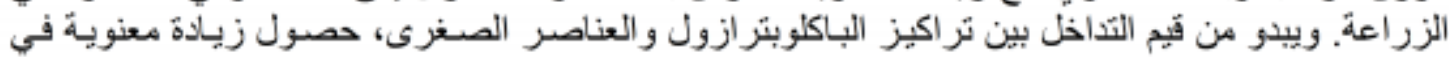

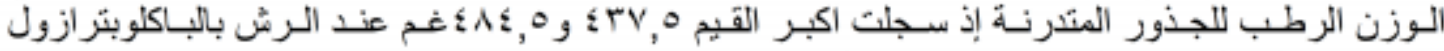

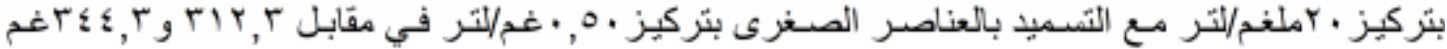

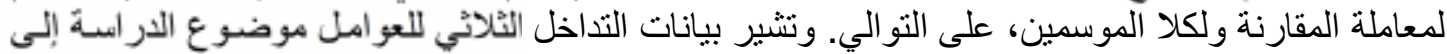

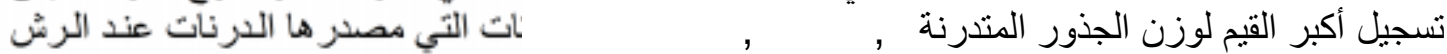

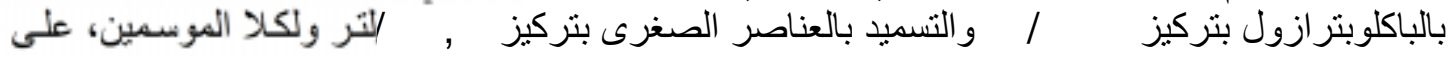

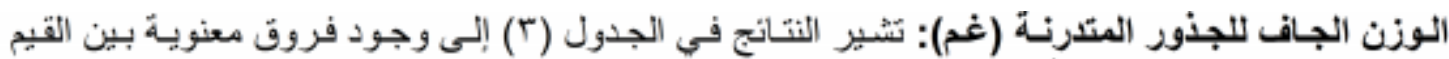

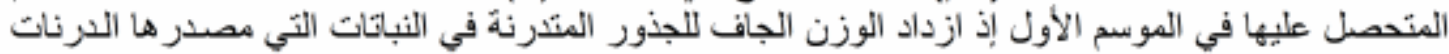

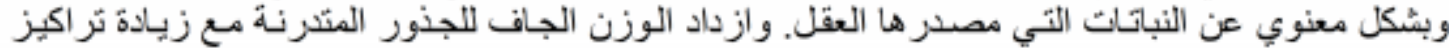

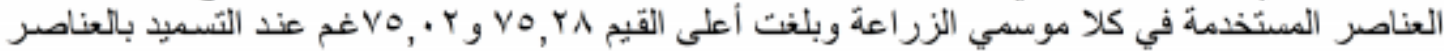

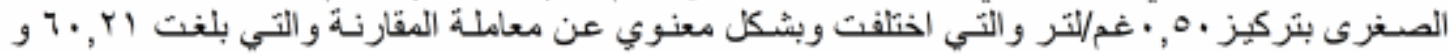

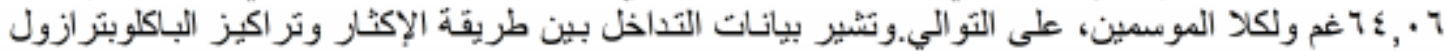

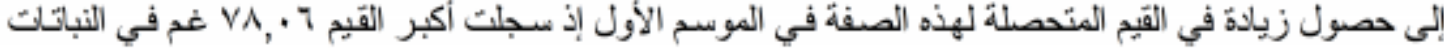

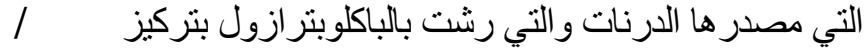

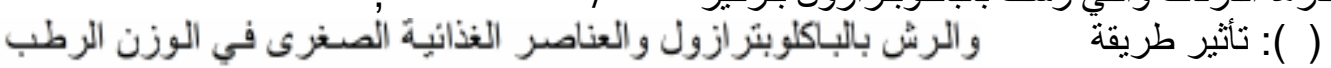

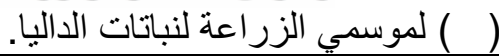

\begin{tabular}{|c|c|c|c|c|c|c|c|}
\hline \multirow{2}{*}{ تأثير طريقة } & \multirow[t]{2}{*}{ تداخل طريقة } & \multicolumn{2}{|c|}{$(1$} & \multicolumn{2}{|c|}{ تراكيز العناصر الصغرى } & تر اكيز & \multirow{2}{*}{ طريقة } \\
\hline & & & & , & & $(1)$ & \\
\hline \multirow{3}{*}{, } & , & & & - & - & & \multirow{3}{*}{ درنات جذرية } \\
\hline & , & & & & - & & \\
\hline & , & & & 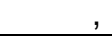 & & & \\
\hline \multirow{3}{*}{, } & , & - & & & $\rightarrow$ & & \multirow{3}{*}{ عقل ساقية } \\
\hline & , & - & & - & ده & & \\
\hline & 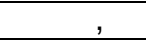 & - & & - & ده & & \\
\hline \multirow{2}{*}{\multicolumn{2}{|c|}{ تأثير الباكلوبترازول }} & 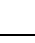 & & & & & \multirow[t]{2}{*}{ تداخل طريقة } \\
\hline & & & & , & $\rightarrow$ & & \\
\hline \multirow{3}{*}{\multicolumn{2}{|c|}{,' }} & - & & & & & \\
\hline & & & & - & & & \\
\hline & & & & - & & & \\
\hline
\end{tabular}



()$\quad(\quad)$
(ISSN 1815-316X)
مجلة زر اعة الر افدين

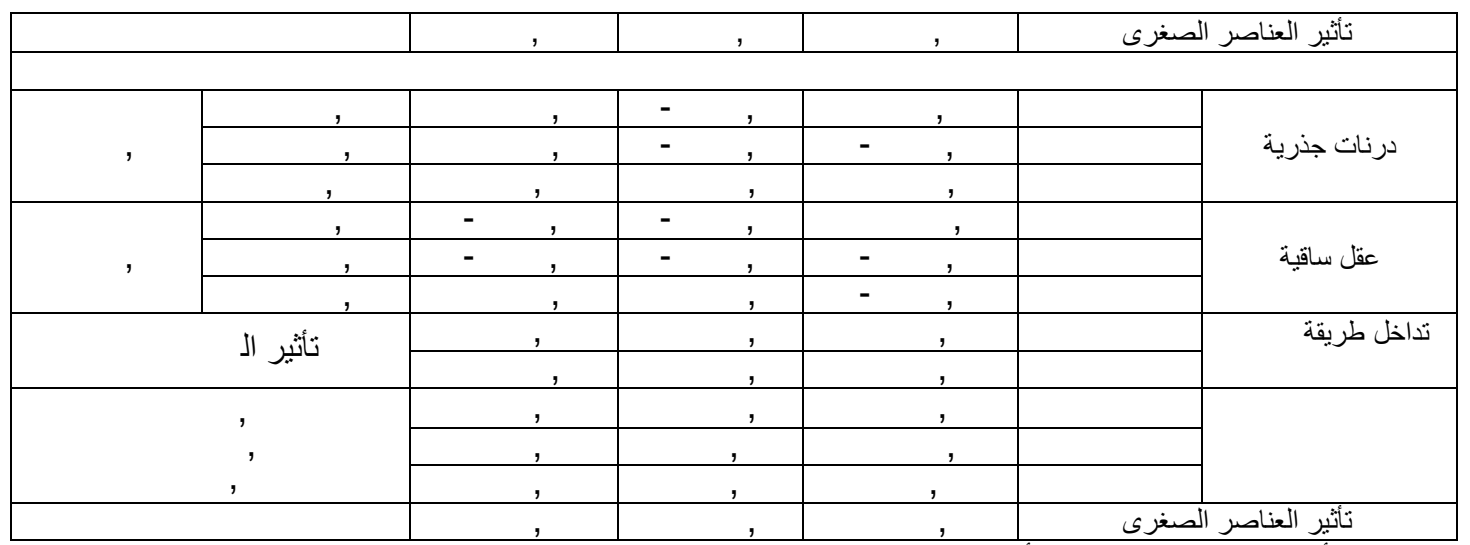

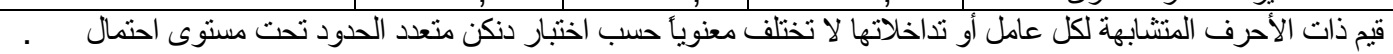

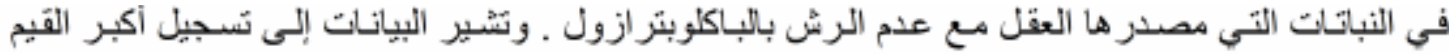

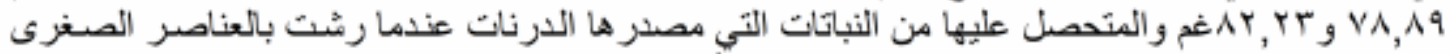

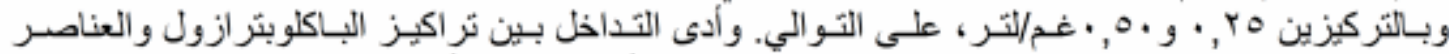

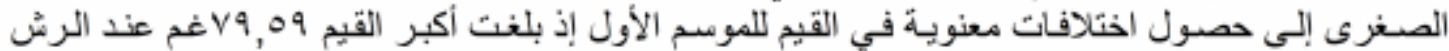

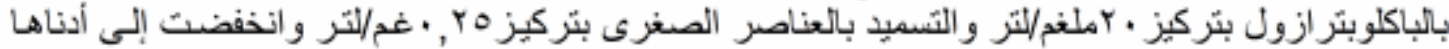

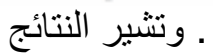

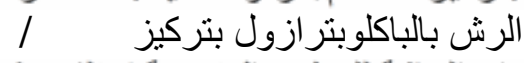

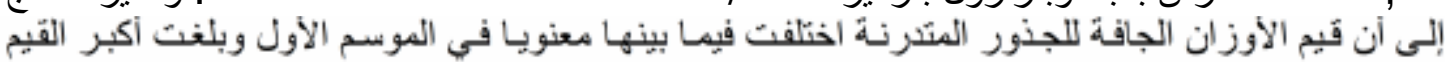

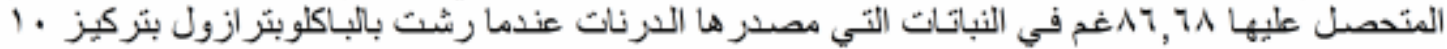

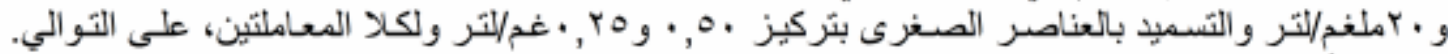

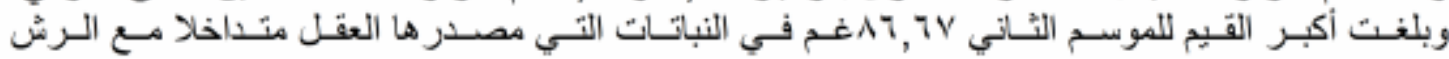

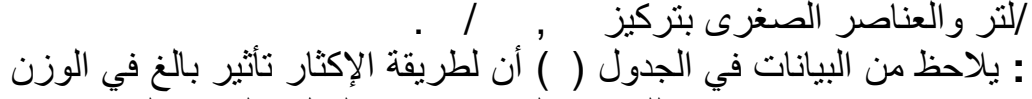

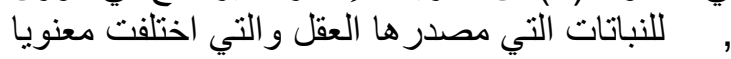

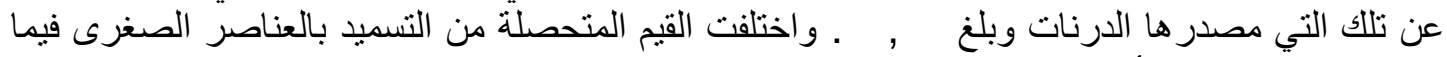

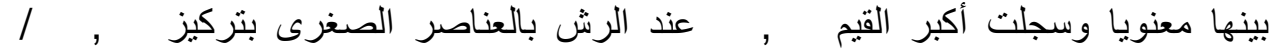

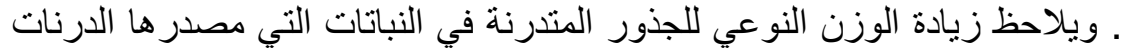

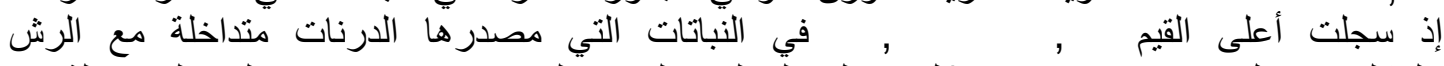

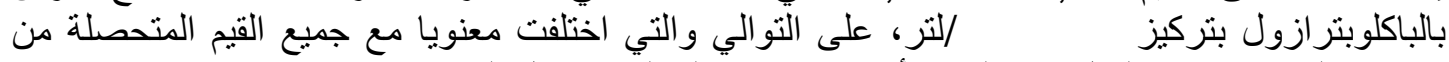

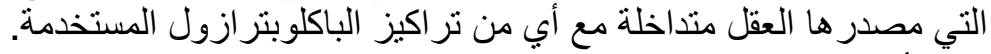

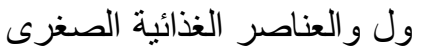

( ) ( ) (أثنير طريقة ل النباتات الداليا.

( )

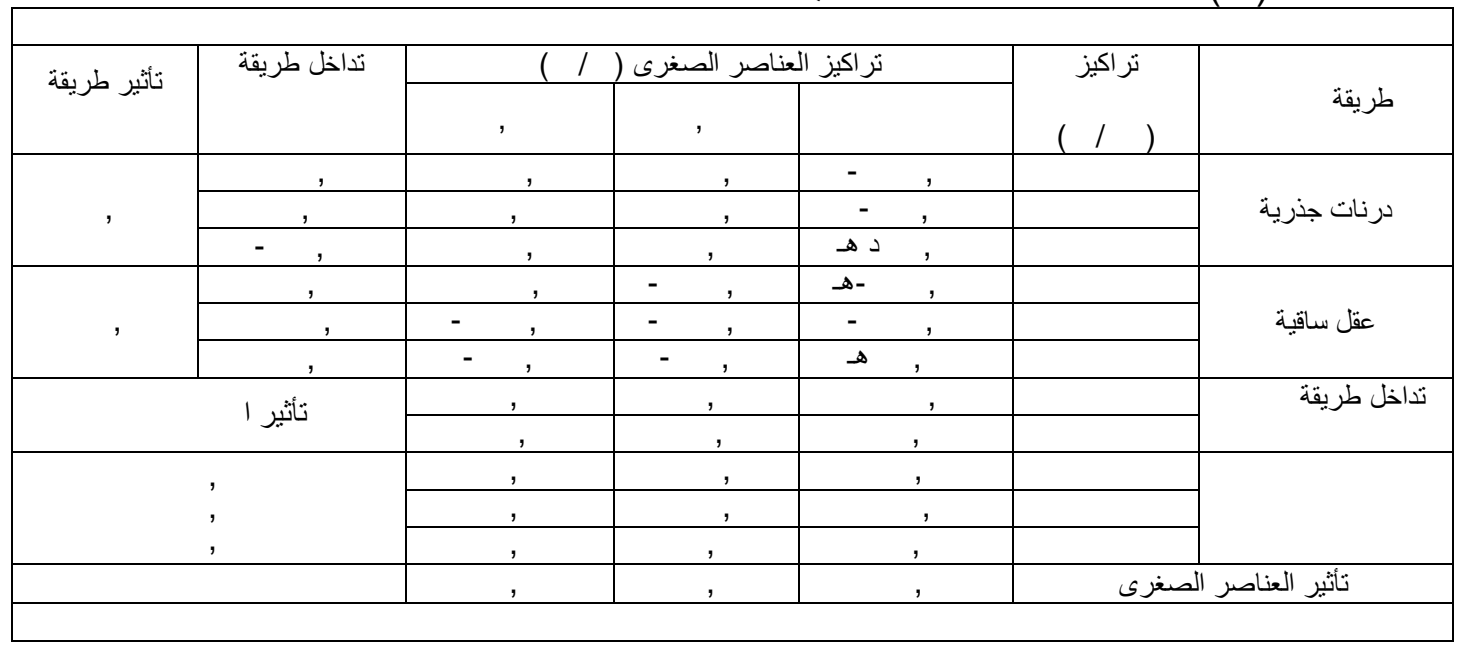



()$\quad(\quad)$
(ISSN 1815-316X)
مجلة زر اعة الر افدين

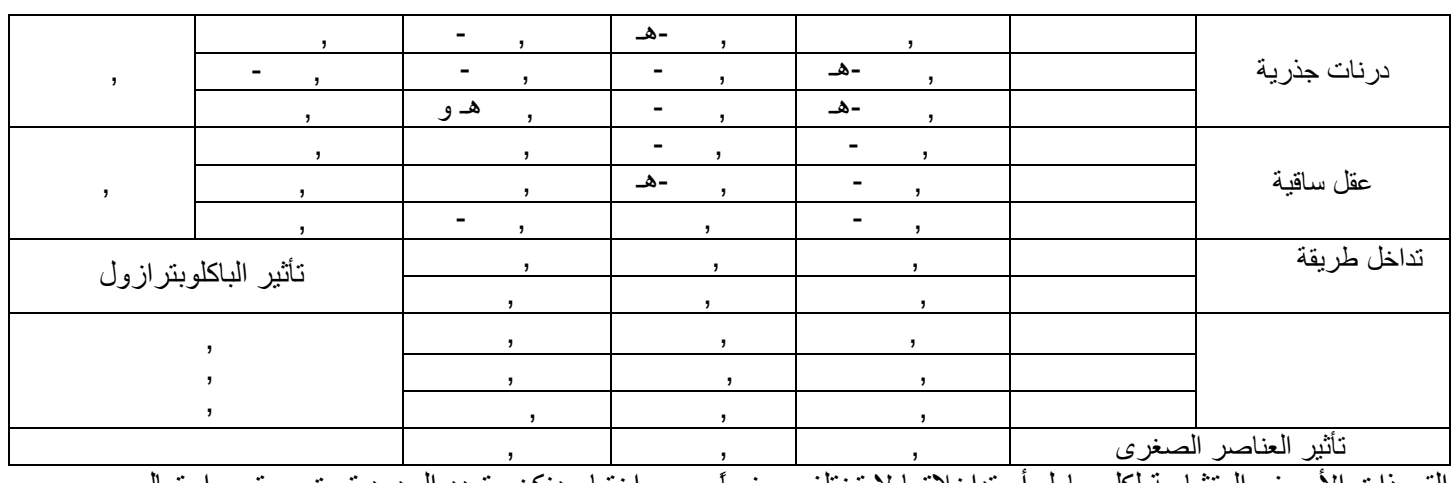

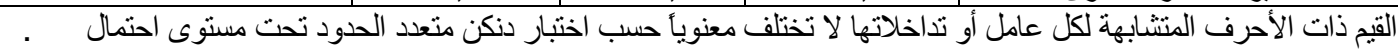

ر القيم للجذور المتدرنة المأخوذة من النباتات التي مصدر ها الدرنات و المسمدة بتركيز

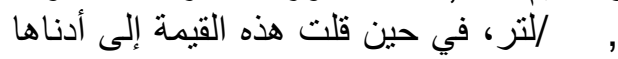

.

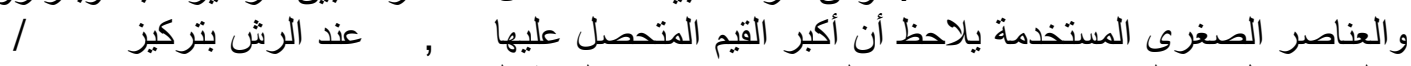

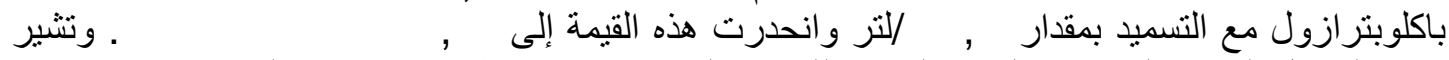

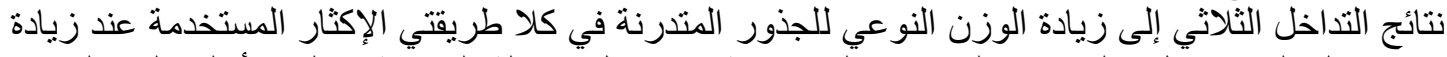

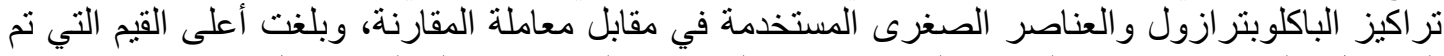

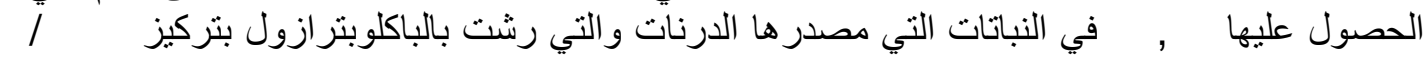

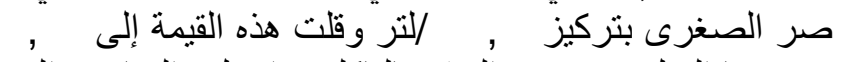

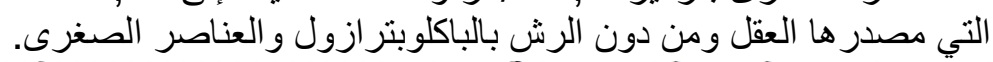

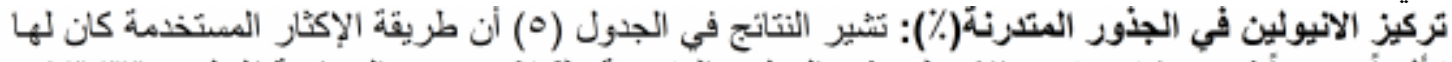

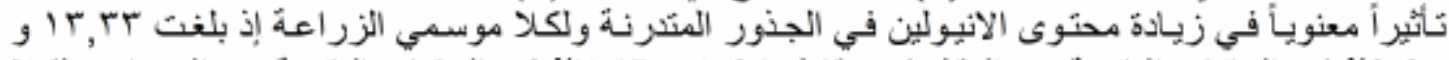

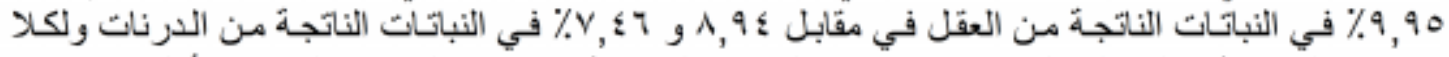

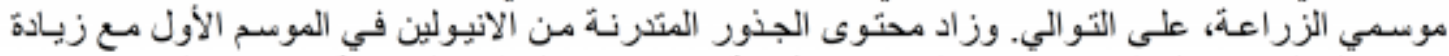

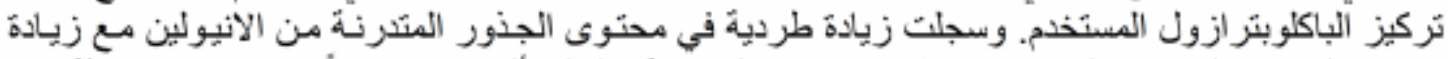

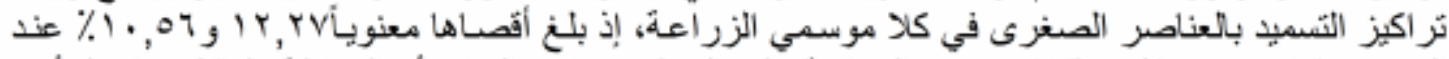

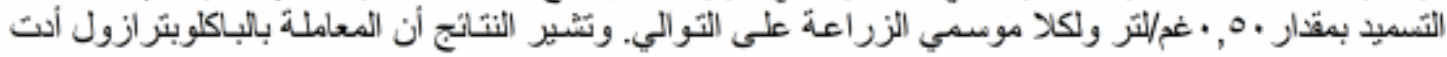

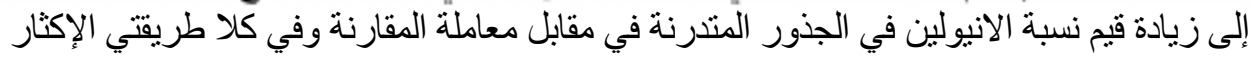

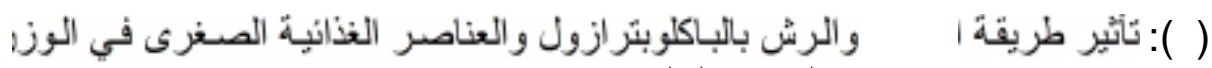

لنباتات الداليا.

\begin{tabular}{|c|c|c|c|c|c|c|}
\hline \multirow{2}{*}{ طريقئة } & \multirow{2}{*}{ طريقة } & \multicolumn{3}{|c|}{ تر اكيز العناصر الصغرى ( / ) ) } & \multirow{2}{*}{$\begin{array}{c}\text { كيز } \\
(\text { / })\end{array}$} & \multirow{2}{*}{ 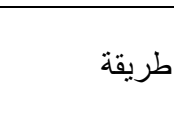 } \\
\hline & & , & 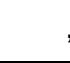 & & & \\
\hline \multirow{3}{*}{, } & , & , & $\rightarrow-$ & - & & \multirow{3}{*}{ درنات جذرية } \\
\hline & , & , & $\rightarrow-$ & $\rightarrow-$ & & \\
\hline & , & , & - & $ه-$ & & \\
\hline \multirow{3}{*}{, } & , & $\Delta-$ & هـ و & & & \multirow{3}{*}{ عقل ساقية } \\
\hline & , & $\Delta-$, & - & هـ و & & \\
\hline & & $-\quad$, & هـ & هـ و & & \\
\hline \multirow{2}{*}{\multicolumn{2}{|c|}{ ت ت تأثير الباكلوبتر ازول }} & 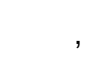 & & & & \multirow[t]{2}{*}{ طريقة } \\
\hline & & , & & & & \\
\hline \multicolumn{2}{|c|}{, } & - & $\rightarrow-$ & & & \\
\hline \multicolumn{2}{|c|}{, } & , & - & $\rightarrow د$ & & \\
\hline \multirow{2}{*}{\multicolumn{2}{|c|}{, }} & , & & ده & & \\
\hline & & & & & 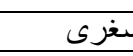 & تأثتير العناص \\
\hline
\end{tabular}


مجلة زر اعة الر افدين

القيم ذات الأحرف المتشابهة لكل عامل أو تداخلاتها تختلف معنوياً حـ

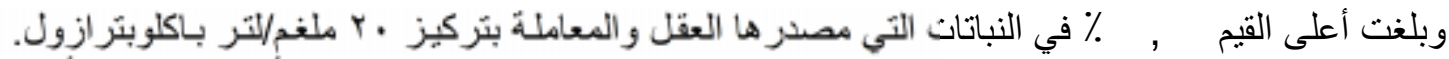

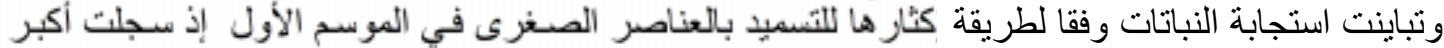

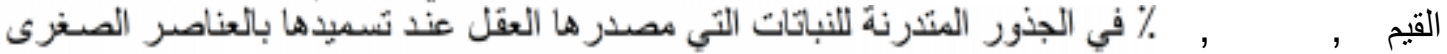

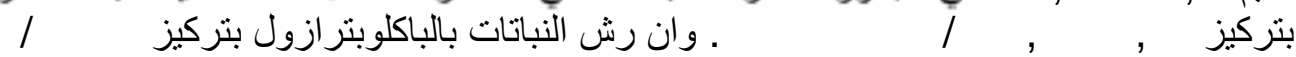

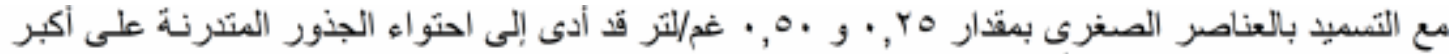

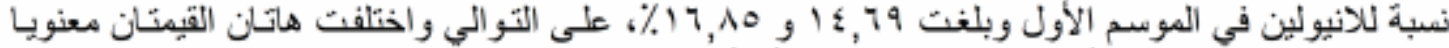

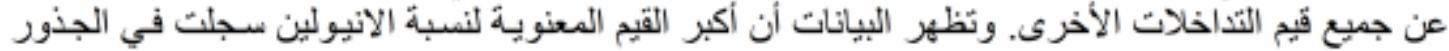

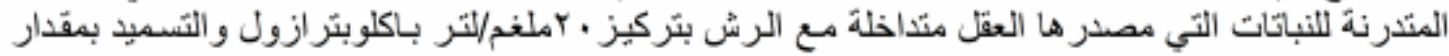
ن الجذور المنّرنة لكل نبات عند استخدام الدرنات الجذريـة في إكثار الثبات الجذول

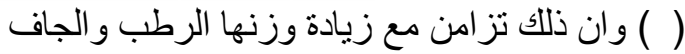

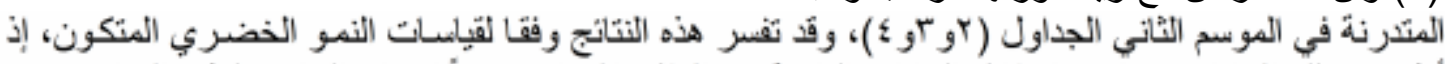

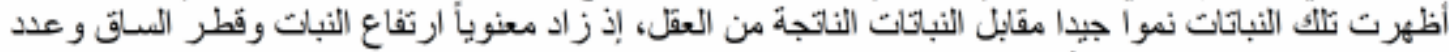

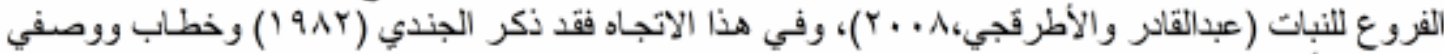
لأل (1911)

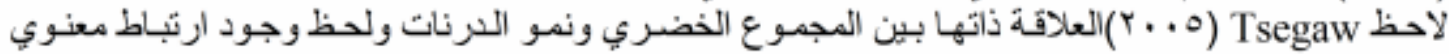

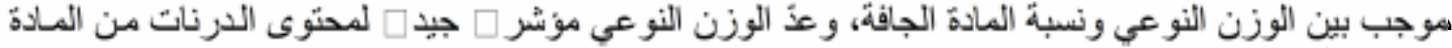

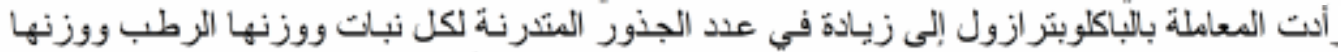

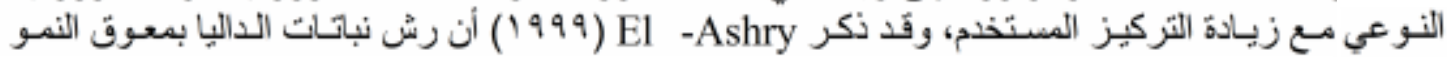

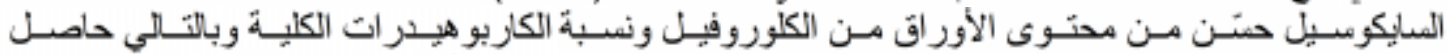

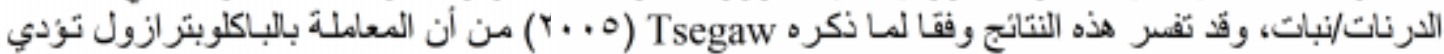

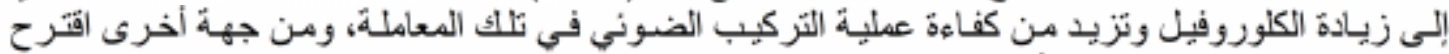

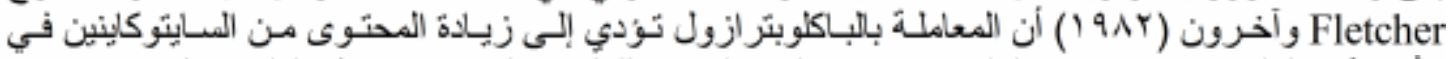

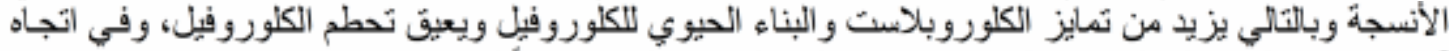

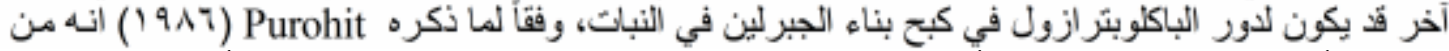

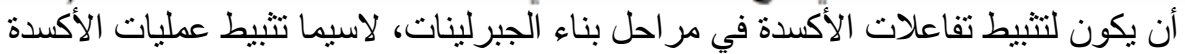

في المايكروسوم لكل Kaurenal Kaurenol Kaurene

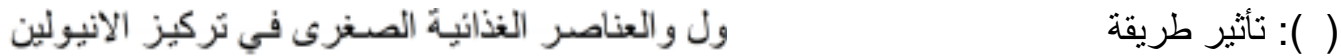

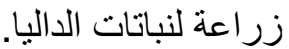

()

\begin{tabular}{|c|c|c|c|c|c|c|}
\hline \multirow{2}{*}{ ت ت تأثير طريقة } & \multirow[t]{2}{*}{ ت تداخل طريقة } & \multicolumn{3}{|c|}{ تراكيز العناصر الصغرى ( / / ) } & \multirow{2}{*}{$\begin{array}{c}\text { تراكيز } \\
\left(\begin{array}{l}1 \\
(\end{array}\right)\end{array}$} & \multirow{2}{*}{ 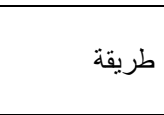 } \\
\hline & & , & , & & & \\
\hline \multirow{3}{*}{, } & & $\Delta$, & $-\quad$, & 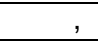 & & \multirow{3}{*}{ درنات جذرية } \\
\hline & , & $-\quad$, & $-\quad$, & , & & \\
\hline & , & , & $\Delta-\quad$, & $-\quad$, & & \\
\hline \multirow{3}{*}{, } & , & & , & - , & & \multirow{3}{*}{ عقل ساقية } \\
\hline & , & $-\quad$, & $\rightarrow-\quad$, & $\Delta-$ & & \\
\hline & , & , & , & $-\quad$, & & \\
\hline \multirow{2}{*}{\multicolumn{2}{|c|}{ تأثيّر الباكلوبترازول }} & 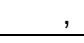 & , & , & & \multirow[t]{2}{*}{ ريقة } \\
\hline & & 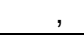 & 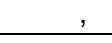 & , & & \\
\hline \multirow{3}{*}{\multicolumn{2}{|c|}{ ', }} & , & , & , & & \\
\hline & & 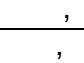 & , & , & & \\
\hline & & 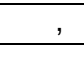 & 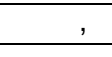 & 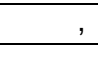 & \multicolumn{2}{|c|}{ تأثير العناصر الصغرى } \\
\hline \multirow[b]{2}{*}{, } & , & , & ه- , , , & $\rightarrow$, & & \multirow{2}{*}{ درنات جذرية } \\
\hline & 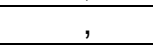 & , & $\rightarrow-\quad$, & $\rightarrow$ & & \\
\hline
\end{tabular}




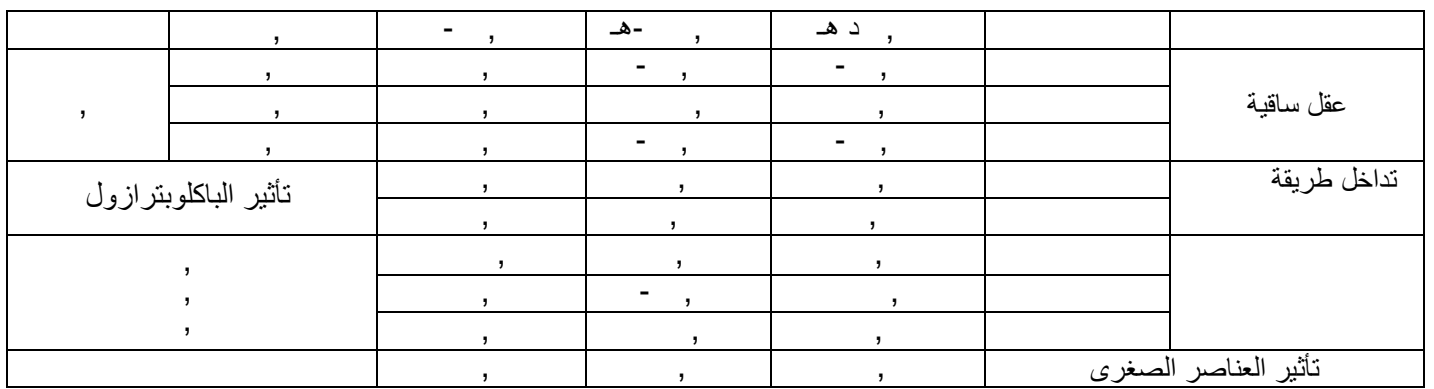

القيم ذات الأحرف المتثابية لكنل عامل أو تداخلاتها لا تختلف معنوياً حسب اختبار دنكن متعدد الحدود تحت مستوى احتمال .

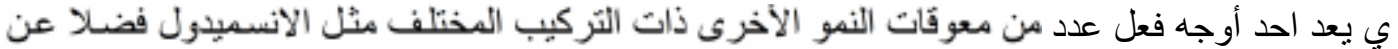

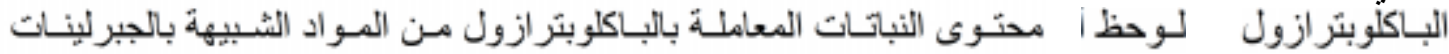

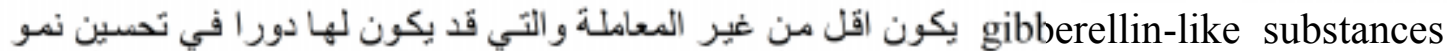
Looth

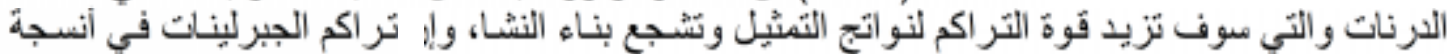

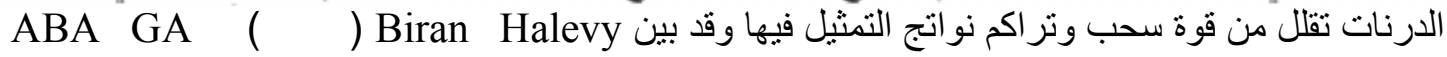

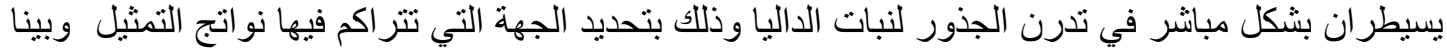

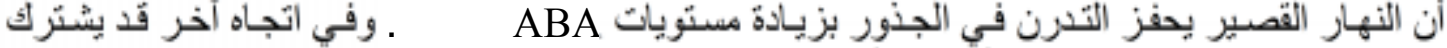

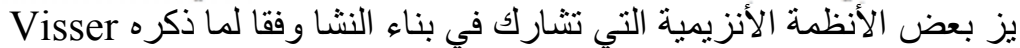

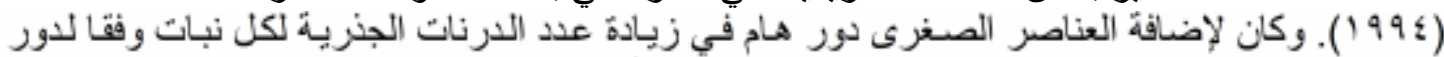

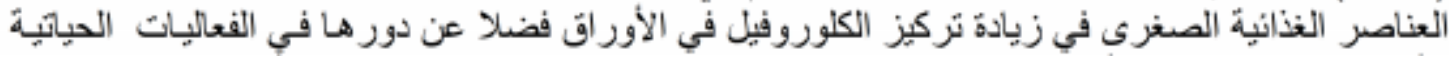
) Ahmed ( ) الغذائبة الصغريى منمثلة Mn B Cu $\quad$ Zn

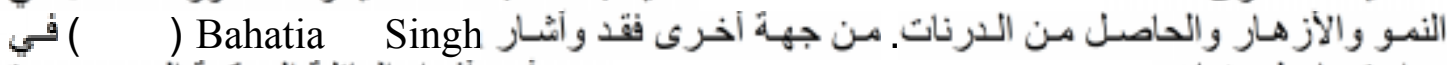

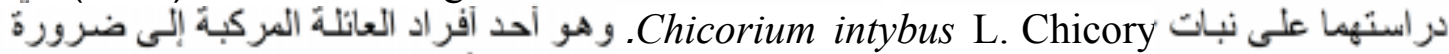

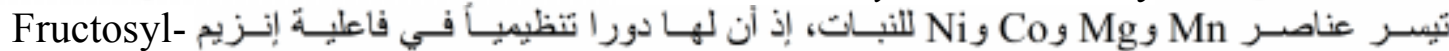

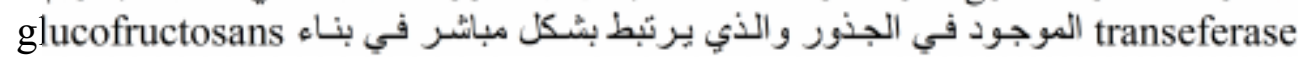

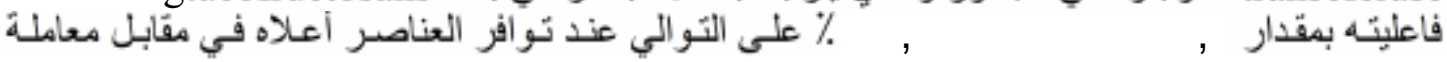

نسبة الانيولين في جذور النباتات الناتجة من العقل كانت اكبر

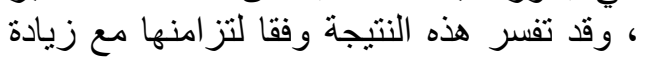

زيادة عملية البناء الضوئي
تشير البيانات في الجدول ( )

من نسبتها في تيزي

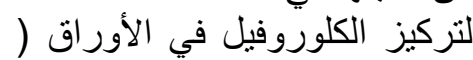
و التي هي مصدر في فئر الكاربو هيدرات

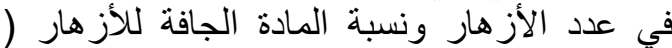
الفائض من المو اد الكاربو هيدراتية قد تر اكم في الجذور بشكل إنيولين لعدم استنز افه في الأزهار ـ ويؤيد ذلك

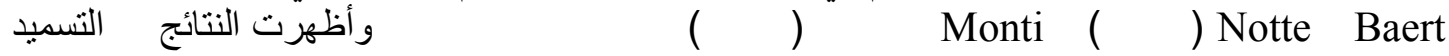
زيادة نسبة الانيولين في الجذور المتدرنة، والذي قد يعود

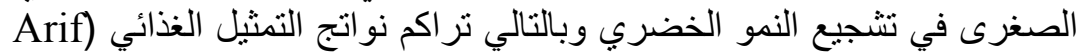

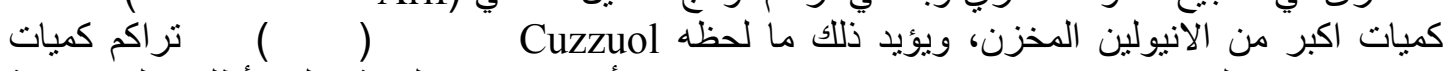
كبيرة من الانيولين في نبات Vernonia herbacea في الأعضاء تحت التربة التبن والتي أطلق عليها تسمية Rhizophores Galiba Kerepesi الكاربون تؤدي إلى تر اكم كميات اكبر من الانيولين

\section{EFFECT OF PROPAGATION METHODS, SPRAYING WITH PACLOBUTRAZOL AND MICRONUTRIENT ON GROWTH OF Dahlia hybrida cv. Edinburgh,}




\title{
3-TUBEROUS ROOTS CHARACTERS
}

\author{
H. A. Abdel-Kadir \\ A. O. Al - Atrakchii
}

Hort. and landscape design Dept., College of Agric. and Forestry, Mosul University

\begin{abstract}
This study was carried on Dahlia hybrida cv. Edinburgh, to compare two propagation methods, application of paclobutrazol PBZ at 0,10 and $20 \mathrm{mg} /$ liter and micronutrients at concentrations $0,0.25$ and $0.50 \mathrm{~g} /$ liter on tuberous roots growth. The results can be summarized as follows: The plants propagated by tuberous roots gave significant increase in roots number, fresh and dry weight and specific weight. But tuberous roots of plants propagated from cuttings contained higher percentage of Inulin 13.33 and $9.95 \%$ dry weight for two seasons, respectively. Tuberous roots taken from plants treated with $20 \mathrm{mg} /$ liter PBZ gave largest number of tuberous roots and heaviest fresh weight, in addition to Inulin percentage.
\end{abstract}

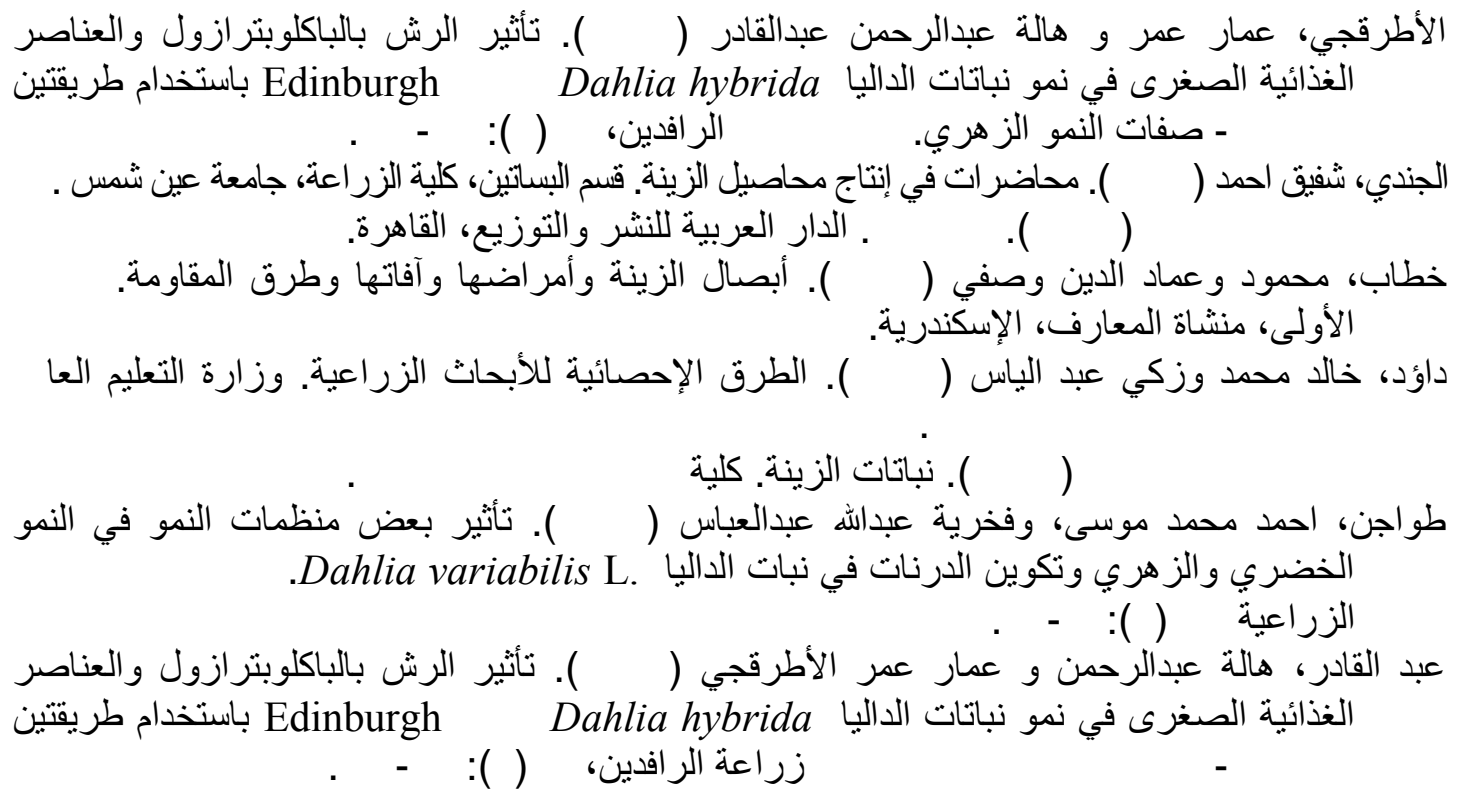

Ahmed, M. , M. Fareed Khan, A. Hamid and A. Hussain (2004). Effect of Urea, DAP and FYM on growth and flowering of dahlia Dahlia variabilis. Int. J. Agric. Biol., 6 (2): 393-395.

Ahmed, M. , M. F. Khan, A. Hamid and A. Hussain (2004). Effect of Urea, DAP and FYM on growth and flowering of dahlia Dahlia variabilis. Int. J. Agri. Biol. 6 (2):393-395.

Arif, M., M.A. Chohan, S. Ali, R. Gul and S. Khan (2006).Response of wheat to foliar application of nutrients. J. Agric. Biol. Sci.,1(4): 30-33.

Baert, J. and Ch. Notte (2002). Effect of selection for bolting resistance on yield and quality of inulin chicory. Ninth Seminar on Inulin. Hungarian Scientific Society for Food Industry: 6-8.

Bajracharya, D. (1999). Experiments in Plant Physiology. Narosa Publishing House. New Delhi. p.50-51.

Bhattacharjee, S.K. and T. Mukherjee (1983). Influence of nitrogen and phosphorus on growth and flowering in Dahlia. Punjab Hort. J. 23:111-115. (Abstract). 
Biran, I., I. Gur and A.H. Halevy (1972). The relationship between exogenous growth inhibitors and endogenous levels of ethylene, and tuberization of dahlias. Physiol. Plant. 27: 226-230.

Booth, A. and P.H. Lovell (1972). The effect of pre-treatment with gibberellic acid on the distribution of photosynthetic in intact and disbudded plants of Solanum tuberosum. New Phytol., 71: 795-804.

Clark, G.E., G.K. Burge (2002). Effects of soilless media pH on cut flower and tuber production in Sandersonia aurantiaca. New Zealand J. of Crop and Hort. Sci., 30: 127-34.

Cuzzuol, G.R.F., M.A.M. Carvalho, C.J. Barbedo and L.B.P. Zaidan (2003). Growth and fructan contents in plants of Vernonia herbacea (Vell.) Rusby cultivated under nitrogen supply. Revista Brasil. Bot. 26(1):81-91.(Abstract).

Dubois, M., K.A. Gilles, J.K. Hamilton, P.A. Rebers and F. Smith (1956). Colorimetric method for determination of sugars and related substances. Anal. Chem., 28(3): 350- 6.

El-Ashry, A.I. (1999). Response of Dahlia pinnata to sprays of growth regulators. Menofiya J. Agric. Res., 24(1): 261-276. (C.F. Al-Humaid, A.I. (2001). Physiological responses of Gladiolus gandavensis cv. Rosesupreme to cycocel (CCC) application. Alex. J. Agric. Res. 46(2): 89-96.

Fletcher, R.A. , V. Kallidumbil and P. Steele (1982). An improved bioassay for cytokinin using cucumber cotyledons. Plant Physiol. 69: 675-677.

Gibson, J.L., P.V. Nelson, D.S. Pitchay and B.E. Whipker (2001). Identifying nutrient deficiencies of bedding plants. NC State University. Floriculture Research. FLOREX, 004: 1-4.

Gupta, A.K. and N. Kaur (1997). Fructan storing plants-A potential source of high fructose syrups. J. Sci. Ind. Res., 56: 447-452. (C.F. Kaur, N. and A.K. Gupta (2002). J. Bio. sci., 27(7): 703-714).

Halevy, A.H. and I. Biran (1975). Hormonal regulation of tuberization in Dahlia. Acta Hort.(ISHS) 47: 319-330.(Abstract).

Hankins, A. (2005). Production of Dahlias as cut flowers. Virginia State University. Virginia Tech. 4( 3):1-4.

Hartmann, H.T., D.E. Kester, F.T. Davies and R.L. Genev (2002). Plant Propagation, Principles and Practices, $7^{\text {th }}$ edition, Prentice Hall, Upper Saddle River, New Jersey.

Kerepesi, I. and G. Galiba (2000). Osmotic and salt stress-induced alteration in soluble carbohydrate content in wheat seedlings. Crop Science 40: 482-487.

Mc Neilan, R. (2000). Dahlia Culture. Oregon State University FS., 95: 1-7.

Monti, A., G. Pritoni and M.T. Amaducci (2002). Evaluation of chicory varieties in north Italy: Inulin production and photosynthesis. Ninth Seminar of Inulin. Hungarian Scientific Society for Food Industry.

Moser, B.C. and C.E. Hess (1969). The physiology of tuberous root development in dahlia. J. Amer. Soc. for Hort. Sci., 93: 595-603.

Mostafa, M.M., I.A. Abou El-Fadi and E.H. Hussein (1996). Effect of phosphorus and boron on the vegetative growth, flowers and corms production and oil yield of tuberose plants. Alex. J. Agric. Res., 41(3): 93-107.

Prasad, R. and J.F. Power (1997). Soil Fertility Management for Sustainable Agriculture. CRC Press LLC. Boca Rotan. New York.

Purohit, S.S. (1986). Hormonal Regulation of Plant Growth and Development, Vol. III. Agro. Botanical Publishers (India). 

( ) ( )
(ISSN 1815-316X)
مجلة زر اعة الر افدين

Rowlands, G. (1999). The Gardener's Guide to Growing Dahlias. Timber Press Inc. North America. Portland, Oregon.

Runger, W. and K.E. Cockshull (1985). Dahlia. In Handbook of flowering vol. II. Halevy, A.H. editor. CRC Press. Boca Raton. Florida: 414-418.

SAS.(1996). Statistical Analysis System, SAS Institute Inc. Cary, NC. U.S.A.

Singh, R. and I.S. Bhatia (1971). Isolation and characterization of fructosyltranseferas from chicory roots. Phytochemistry, 10: 495-502.

Singh, Z. and A.K. Gupta (1995). Effect of nitrogen, phosphorus and potassium on tuber production in Dahlia variabilis Wills cv. Powder puff. Crop. Res., 10(2): 174-178.

Stevens, A.B. (1995). Fertilization of field grown specialty cut flower. Kansas State University. Cooperative Extension Service, MF-2154: 1-8.

Syverson, D. (2002). The Role of soil fertility in gardening. Minnesota Dahlia Society: 1-4.

Tsegaw, T. (2005). Response of potato to paclobutrazol and manipulation of reproductive growth under tropical conditions. Ph.D. Thesis. Dept. of plant production and soil science. Pretoria University.

Vijn, I. and S. Smeekens (1999). Fructan: more than a reserve carbohydrate. Plant Physiol., 120: 351-359.

Visser, R.G.F., D. Vreugdenhil, T. Hendrix and E. Jacobsen (1994). Gene expression and carbohydrate content during stolon to tuber transition in potatoes Solanum tuberosum L. . Physiol. Plant., 90: 285-292. 